\title{
Efectos de la aplicación intrahipocampal del péptido BLMP-101 sobre una tarea de memoria espacial en ratas Wistar ${ }^{*}$
}

\section{Effects of Intrahypocampal Administration of the BLMP-101 Peptide on a Spatial Memory Task in Wistar Rats}

Recibido: noviembre 22 de 2009 ｜ Revisado: febrero 20 de 2010 | Aceptado: marzo 2 de 2010

\author{
LEONARDO LAREO ** \\ JosÉ ARTURO BRIÑEZ-HORTA **** \\ RAÚl OYUELA-VARGAS ${ }^{* * * * *}$ \\ SONIA ALBARRACÍN ${ }^{* * * * *}$ \\ Pontificia Universidad Javeriana, Bogotá Colombia \\ LAURA ANDREA-LEÓN ${ }^{* * * * * * *}$ \\ FERNANDO P. CARDENAS \\ Universidad de los Andes, Bogotá, Colombia
}

Para citar este artículo. Laero, L., Bríñez Horta, J.A., Oyuela Vargas, R., Albarracín, S., León, L.A. \& Cardenas, F.P. (2010). Efectos de la aplicación intrahipocampal del péptido BLMP-101 sobre una tarea de memoria espacial en ratas Wistar. Universitas Psychologica, 9 (3), 679-688.

* Resultado del proyecto de investigación "Aprendizaje y memoria de trabajo en función de la edad, en la rata Wistar", financiado por la Pontificia Universidad Javeriana (registro 000070).

** Facultad de Ciencias. Cr. 7 \# 43-82, Bogotá, Colombia. Correos electrónicos: 1.lareo@javeriana. edu.co

**** Laboratorio de Psicología. Cr. 5 \# 39-00 oficina 14. Tel: 3208320 ext. 5711. Correo electrónico: artubrinez@gmail.com

****** Facultad de Psicología. Av. Cra. 68 \# 22A-30. Correo electrónico: raul.oyuela@gmail.com

******* Facultad de Ciencias. Cr. 7 \# 43-82, Bogotá, Colombia. Correos electrónicos: albarra@javeriana. edu.co

********* Facultad de Psicología. Cr. 1 \# 18A-10 ed. Franco, piso 2, Bogotá, Colombia. Correos electrónicos: la-leon@uniandes.edu.co; lucarden@uniandes. edu.co

RESUMEN

El péptido BLMP-101 posee potencial para el tratamiento de deficiencias de la memoria. Esta investigación tuvo como objetivo corroborar los resultados obtenidos en investigaciones anteriores con respecto a la evaluación del péptido BLMP-101, en cuanto a la efectividad del péptido diseñado por el grupo de Bioquímica Computacional y estructural de la Pontificia Universidad Javeriana. Sin embargo, y a diferencia de las investigaciones anteriores, el objetivo del presente estudio consistió en medir la efectividad del BLMP-101, en dosis diferentes y haciendo la aplicación intrahipocampal del péptido, para corroborar sus efectos en la facilitación de la memoria espacial. Se utilizaron 31 ratas Wistar divididas en cuatro grupos, tres experimentales y uno de comparación. El grupo de comparación, fue inyectado con solución salina y a uno de los grupos experimentales se le administró NMDA y, a los otros dos, dosis diferentes del péptido BLMP-101 (0.3, 3.0 ug/ul). Los resultados sugieren que el péptido BLMP-101 con dosis de 3.0 ug/ul, facilitan la memoria espacial, mejor que el NMDA.

Palabras clave autores

Péptido (37330); Aprendizaje (28030); Aprendizaje en ratas (42860).

Palabras clave descriptor

Péptidos, aprendizaje, ratas, psicología.

\begin{abstract}
A B S T R A C T
The BLMP-101 peptide has the potential features to treat memory deficiency. The herein research aimed at corroborating the results obtained in previous researches and tests conducted on the effectiveness of the peptide BLMP-101 designed by the Computational and Structural Biochemestry Unit of the Pontificia Universidad Javeriana, Colombia. However, and unlike previous researches, the objective of this paper measured the effectiveness of the BLMP-101peptide, when supplied in different dozes and through intrahipocampal application to confirm its effects to facilitate spatial memory. 31 Wistar rats were used into 4 different groups: three treatment groups
\end{abstract}


and one control group. The control group was infused with saline solution, and one of the treatment groups was infused with NMDA, while the other two experimental groups were infused with different dozes of the BLMP-101peptide (0.3, $3.0 \mathrm{ug} / \mathrm{ul})$. Results suggest that the BLMP-101 peptide, when supplied in $3.0 \mathrm{ug} / \mathrm{ul}$, facilitates spatial memory showing better results than the NMDA.

Keywords authors

Peptyde (37330); Learning (28030); Learning in Rats (42860).

Keywords plus

Peptides, Learning, Rats, Psychology.

El receptor ionotrópico de glutamato activado por $\mathrm{N}$-metil-D-aspartato (iGluR-NMDA) es, sin lugar a duda, uno de los complejos macromoleculares más polifuncionales del sistema nervioso central (SNC) (Heresco-Levy \& Javitt, 1998). Algunos de los procesos cognitivos, aprendizaje y memoria, requieren sin equa non de la participación directa del iGluR-NMDA, para que puedan ocurrir (Riedel, Platt \& Micheau, 2003; Tang, Shimizu, Dube, Rampon, Kerchner, Zhuo, Liu \& Tsien 1999). Este hecho ha abierto la puerta de las oportunidades, para el desarrollo de sustancias que permitan incrementar, o al menos solo mantener, los niveles normales de desempeño cognoscitivo, particularmente durante el desarrollo de las enfermedades neurodegenerativas. Inclusive desde antes de las demostraciones claras del grupo de Tsien se habían hecho intentos en este sentido (Allain, Lieury, Reymann, Martinet, Trebon, Decombe, BentueFerrer \& Gandon, 1990) y el interés en el tema se ha mantenido (Narahashi, Moriguchi, Zhao, Marszalec \& Yeh, 2004).

Luego de las demostraciones de que las toxinas de los caracoles marinos del género Conus, en especial las llamadas conantoquinas, interactúan específicamente con el receptor iGluR-NMDA (Haack, Rivier, Parks, Mena, Cruz \& Olivera, 1990; Hammerland, Olivera \& Yoshikami, 1992) y de que esto ocurre también en humanos (Nielsen, Skjaerbaek, Dooley, Adams, Mortensen, Dodd, Craik, Alewood \& Lewis, 1999), además del avance en el reconocimiento de que esta interacción inhibitoria es selectiva para la subunidad NR2B, constitutiva del iGluR-NMDA (Donevan \& McCabe, 2000), la cual estaba ya demostrada como esencial en los procesos de aprendizaje y memoria (Tang et al., 1999; Tang, Wang, Feng, Kyin \& Tsien, 2001), se ratificó la posibilidad de diseñar moléculas exógenas para modificar los procesos cognitivos de interés. Con base en la comprensión de aspectos estructurales publicados (Blandl, Warder, Prorok \& Castellino, 2000; Blandl, Zajicek, Prorok \& Castellino 2001) y siguiendo la tendencia mundial empleada para otros propósitos, de derivar drogas a partir de productos naturales (Alonso, Khalil, Satkunanthan \& Livett, 2003; Ault, 2004), asî como las guías dadas para el diseño de drogas en el futuro cercano para mejorar la cognición, especialmente en los casos de demencias, hechas por van Reekum (Van Reekum, Black, Conn \& Clarke, 1997), se diseñó de novo y sintetizó un conjunto de péptidos (Oyuela, Lareo, Muñoz, Morales, Echeverri, Uribe, Santos \& Acuña, 2004) que potencialmente debería incrementar los procesos de aprendizaje y memoria, lo cual fue demostrado en modelos animales (Oyuela et al., 2004), y se ha ratificado en diferentes trabajos a partir de ese momento (Bernal, Rojas \& Oyuela, 2006; Echeverry \& Oyuela, 2006; Santacruz, Oyuela, Bríñez, Lareo $\&$ Echeverri, 2007).

De hecho, dentro de los modelos tradicionalmente utilizados para el estudio general de la memoria se cuentan: el laberinto acuático de Morris, el laberinto radial, las cajas de evitación, entre otros. Actualmente uno de los modelos más empleados en el estudio particular de la memoria espacial, ha sido el test de Barnes (1979), el cual utiliza una plataforma radial con doce agujeros ubicados en su periferia y una caja refugio localizada bajo uno de estos agujeros. La tarea consiste en aprender la ruta que conduce desde el punto de inicio hasta la caja meta, labor que demanda la elaboración de un mapa espacial que lleve a la rata hacia el sitio seguro.

Barnes (1979) mostró que, al estimular el giro dentado y la vía perforante, en ratas, éstas exhibían una menor capacidad para recordar el lugar en donde estaba la caja habitat, que en esta situación, constituye un refuerzo, debido a la aversividad que caracteriza a la superficie. Así, este modelo puede ser utilizado como un modelo confiable para medir 
memoria espacial. Por su parte, Burke, Chawla, Penner, Crowell, Worley, Barnes y McNaughton (2005), explican que el laberinto radial le permite a la rata ubicar claves contextuales en las cuales el movimiento se da, pero no evalúa la ubicación espacial (y como consecuencia la memoria espacial) per se. Una de las ventajas del laberinto de Barnes, en relación con el laberinto de Morris, es el hecho de que en el caso del Morris, el ambiente acuático puede, además de inducir niveles altos de estrés, ocasionar infecciones en ratas previamente sometidas a cirugía, factores que disminuyen la confiabilidad de los datos obtenidos. Para el buen funcionamiento de los procesos mnésicos es indispensable hacer referencia a las diferentes fases que los componen: adquisición, almacenamiento y evocación. La primera constituye la etapa en la que la información ingresa al sistema y es codificada para su posterior consolidación. El almacenamiento hace alusión al mantenimiento de los datos a corto, mediano o largo plazo para su utilización. Por último, la evocación se refiere al acceso a la información previamente almacenada.

Debido a que el papel de los receptores NMDA sobre el aprendizaje tiene lugar en las primeras fases del mismo y dado el efecto agonista del péptido sobre estos receptores, el presente estudio se centrará sobre la etapa de adquisición. Sin embargo, es importante aclarar que muchas sustancias exógenas, que actúan como agonistas o antagonistas del receptor NMDA, pueden modular los procesos cognoscitivos, en especial los de aprendizaje y memoria (Blandl, Zajicek, Prorok \& Castellino, 2001).

En efecto, la modulación de procesos cognoscitivos, como aprendizaje y memoria, por agentes externos, continúa siendo un reto y un campo abierto de oportunidades, para la investigación tanto básica como aplicada. El solo hecho de que existan más de 1.000 patentes de sustancias que se han desarrollado para lograr esa modulación, generalmente incrementado las capacidades reducidas por alguna patología, usualmente neurodegenerativa, revela el interés mundial en el tema. Luego de los hallazgos de Tsien y Ezzel (2000), en la Universidad de Princeton, que demostraron en forma categórica el papel indispensable del receptor ionotrópico de glutamato activado por N-metil-D-aspartato (iGluR-NMDA) en los procesos de aprendizaje y memoria, se abrió una nueva posibilidad para intentar modular estos procesos a través de sustancias desarrolladas, para que interactúen específicamente con dicho receptor.

Por su parte, en la Pontificia Universidad Javeriana se ha diseñado, sintetizado y evaluado un péptido, denominado BLMP-101 (Boost Learning Memory Peptyde-101), que ha resultado, en experimentos realizados previamente, ser una sustancia promisoria logrando los efectos de mejorar la capacidad de aprendizaje y memoria en roedores. En trabajos previos, se han realizado administraciones intraperitoneales del péptido (Lareo, Oyuela, Muñoz, Morales, Echeverri, Uribe, Santos \& Acuña, 2005; Oyuela, Lareo, Muñoz, Morales, Echeverri, Uribe, Santos \& Acuña. 2004; Santacruz, Oyuela, Bríñez, Lareo \& Echeverri, 2008), lo que ha permitido incrementar el conocimiento sobre el funcionamiento de la sustancia. En este sentido, el objetivo de la presente investigación consiste en determinar el efecto de la aplicación intrahipocampal de diferentes dosis del péptido BMLP-101, sobre la memoria espacial, en ratas Wistar.

\section{Método}

\section{Diseño}

El diseño consistió en dividir 31 sujetos en cuatro grupos; tres experimentales y uno de comparación. A uno de los grupos experimentales se le asignaron 7 sujetos, a otro 8 y al tercer grupo 9 sujetos. Por su parte, al grupo de comparación, el cual fue inyectado con solución salina, se le asignaron 7 sujetos. A uno de los grupos experimentales se le administró NMDA y a los otros dos, dosis diferentes del péptido BLMP-101 (0.3, 3.0 ug/ul). En cuanto al diseño y análisis estadístico, éste se realizó mediante ANOVA multivariante, dado que se satisfizaron los supuestos de normalidad y de homogeneidad de varianzas, aunque no se satisfizo la igualdad de las matrices de varianza-covarianza. 


\section{Sujetos}

Fueron utilizadas 31 ratas albinas, Wistar, machos, provenientes del bioterio del Instituto Nacional de Salud, que pesaban en promedio $290 \mathrm{~g}$ en el momento de la cirugía. Los sujetos fueron alojados por parejas. Después de la cirugía, fueron colocados en jaulas individuales con temperatura controlada $\left(22 \pm 2^{\circ} \mathrm{C}\right)$ en ciclo claro-oscuro de 12 horas, con las luces prendidas a las 7:00 a.m. y con presión negativa para el control séptico. Además, se garantizó que los animales utilizados se mantuvieran con agua y comida (Rodentina) ad libitum, en tanto las condiciones experimentales lo permitieran. Además, durante la realización de esta investigación, se cumplieron las normas éticas y legales exigidas para la investigación con animales de laboratorio en Colombia (Ley 84 de 1989 y Resolución No. 8430 de 1993 del Ministerio de Salud).

\section{Instrumentos}

\section{Test de memoria de Barnes}

El instrumento utilizado para evaluar las sustancias administradas en la memoria espacial de los sujetos fue el Test de memoria de Barnes. Este test, permite evaluar la memoria espacial en términos de la latencia y los errores cometidos, por los sujetos. El test de memoria de Barnes consiste en una plataforma redonda (1,20 $\mathrm{m}$ de diámetro) de superficie blanca, ubicada en un cuarto bien iluminado. En la periferia de la plataforma existen 12 agujeros de $10 \mathrm{~cm}$ de diámetro cada uno, colocados a espacios iguales. Debajo de uno de ellos hay una caja que sirve como refugio para el sujeto.

\section{Sustancias}

En cuanto a las sustancias, se utilizó solución salina, NMDA y el péptido BLMP-101. Fueron utilizadas dos dosis del péptido BLMP-101: 0.3 y $3.0 \mathrm{ug} / \mathrm{ul}$; el péptido fue disuelto en solución salina (0,09\%). NMDA: (0.2 ug/ul) fue igualmente disuelto en solución salina.

\section{Procedimiento}

El procedimiento se estructuró en cuatro etapas: cirugía, microinyección de las sustancias, evaluación de la memoria espacial en el test de Barnes e histología.

\section{Cirugía}

Para el implante de las cánulas en hipocampo ventral, los animales fueron inyectados intraperitonealmente con una mezcla de Ketamina y Xilacina (75 mg/kg y $10 \mathrm{mg} / \mathrm{kg}$ respectivamente), colocados en un aparato estereotáxico e inyectados con pentabiótico veterinario (80.000 UI). Fueron realizados orificios bilaterales, para permitir la llegada de la cánula a las siguientes coordenadas: $\mathrm{AP}=3.7$ $\mathrm{mm}$ (con relación a interaural), $\mathrm{ML}= \pm 4.6 \mathrm{~mm}$ y $\mathrm{DV}=5.0 \mathrm{~mm}$, de acuerdo con el atlas de Paxinos y Watson (1997). Se realizaron dos orificios más pequeños, para colocar tornillos para anclar las cánulas. Después de la colocación, las cánulas fueron fijadas con cemento acrílico. Terminada la cirugía, los animales eran llevados nuevamente a sus cajas, donde permanecieron seis días en recuperación. Posterior a estos seis días, después de la cirugía, los 31 sujetos fueron divididos en los cuatro grupos.

\section{Microinyección}

La microinyección fue realizada a través de una aguja dental de una longitud de un milímetro superior a la de la cánula guía. La aguja estaba unida a una jeringa de Hamilton, por medio de un catéter P-10. La aplicación de la sustancia fue realizada a una tasa de 1 ul por minuto. Terminada la microinyección, la aguja se dejó en el lugar durante un minuto más, para evitar la difusión de la sustancia.

\section{Test de memoria de Barnes}

Para habituar los sujetos a la plataforma, estos fueron colocados inicialmente en la caja meta, durante un minuto. Luego, fueron colocados en la plataforma justo encima de la caja meta y se les 
ayudó a entrar en la caja meta. Después de la fase de habituación, se realizó la sesión de entrenamiento 1, en la cual los sujetos eran colocados en el centro de la plataforma, dentro de la caja de inicio, orientada hacia el agujero donde se encuentra la caja meta y se les permitía que exploraran la plataforma hasta encontrar la caja meta, durante 180 segundos. Si pasado ese tiempo, el sujeto no encontraba la caja, se le señalaba la dirección correcta. Después de que el sujeto entraba en la caja meta, se le permitía permanecer allí por 2 minutos. Veinticuatro horas después, las ratas eran colocadas nuevamente en el centro de la plataforma, dentro de la caja de inicio con una orientación aleatoria con relación a la ubicación de la caja meta. La caja de inicio era entonces retirada y se permitía que el sujeto explorara la plataforma hasta encontrar la caja meta que se hallaba en una nueva posición, o hasta que transcurran 180 segundos. Los sujetos eran entonces devueltos a la caja habitat, donde permanecían por quince minutos. Durante ese tiempo, el laberinto era limpiado con una solución de etanol al 20\% y rotado para evitar pistas olfativas. Luego de la fase de introducción de la nueva posición (Ensayo 0), la caja meta era mantenida en la misma posición durante los siguientes ocho ensayos. Los Ensayos 0 a 4 se realizaron el primer día y los Ensayos 5 a 8 el segundo día. El tiempo para llegar a la caja meta y el número de errores, fueron contabilizados. Los errores fueron definidos como olfateo de agujeros incorrectos. La microinyección de las sustancias se realizó inmediatamente antes del ensayo 5. Todas las sesiones fueron registradas por una cámara conectada a un computador en un cuarto adyacente.

\section{Histología}

El estudio histológico tuvo dos objetivos. Por un lado, determinar la correcta colocación de la cánula en la estructura deseada y, por otro, evaluar la posible inducción de modificaciones a largo plazo en la ramificación dendrítica hipocampal. Para la determinación de la correcta colocación de la cánula, los cortes fueron coloreados con técnica de Nissl de violeta de cresilio. Para la evaluación de posibles efectos a largo plazo sobre el proceso de ramificación dendrítica, se realizó la marcación para proteína asociada a microtúbulos MAP2 (Johnson \& Jope, 1992). Para la realización de las coloraciones, la mitad de los sujetos de cada grupo fue perfundida transcardíacamente, inmediatamente terminado el ensayo en el laberinto de Barnes. La otra mitad de cada grupo permaneció en las cajas habitat por un período de 8 días más, antes de ser igualmente perfundidos. La perfusión transcardíaca se realizó con $100 \mathrm{ml}$ de solución salina (0.9\%), seguidos de $300 \mathrm{ml}$ de paraformaldehído (4\%). Los cerebros fueron retirados y dejados en paraformaldehído, durante al menos cuatro días a $4 \stackrel{\circ}{ } \mathrm{C}$. Fueron realizados cortes de 40 um de espesor en un vibrátomo (Vibratome 1500).

El análisis de la ramificación dendrítica, se realizó calculando la relación entre el área ocupada por las fibras inmunomarcadas para MAP2 y el área total del campo. Se utilizó un análisis de varianza ANOVA de una vía. Cuando fue necesario, se realizaron comparaciones de las medias de los grupos utilizando el test post hoc Student Newman-Keuls. El nivel de alfa fue estipulado en 0.05. Los cortes tratados con coloración de Nissl, fueron utilizados para seleccionar los animales que entraron en el estudio; animales con cánulas en lugares diferentes al hipocampo ventral, fueron retirados del estudio.

\section{Consideraciones éticas}

Durante la realización de este proyecto, se cumplieron las normas éticas y legales exigidas para la investigación con animales de laboratorio en Colombia (Ley 84 de 1989 y Resolución No. 8430 de 1993 del Ministerio de Salud). Por razones obvias, este tipo de experimentos sólo se puede realizar en animales de laboratorio, como ratas. Se garantizó que los animales que se utilizarían se mantuvieran con agua y comida (Rodentina) ad libitum, en tanto las condiciones experimentales así lo permitieron.

En este proyecto se requirieron mínimas cantidades de fármacos, los cuales se manipularon y descartaron siguiendo procedimientos de bioseguridad ya establecidos en el laboratorio. Cabe aclarar que 
los reactivos utilizados son de categoría no tóxica, por lo tanto, el estudio no provocó ninguna alteración en el medio ambiente.

\section{Resultados}

La Figura 1 muestra los promedios de latencias de respuesta de resolución del laberinto de Barnes para los animales de todos los grupos, para los Ensayos 3 a 10, y el ensayo de test. Los análisis independientes de latencias y número de errores por ensayos, para los Ensayos 4 y 5, en los cuales se observa la inflexión de la curva de aprendizaje, así como la delta entre los ensayos cuatro-test y cinco-test se representan en las figuras siguientes. El ANOVA mostró diferencias significativas $\left(F_{[3,27]}=4.038 ; P=0.017\right)$ para la latencia de respuesta durante el Ensayo 5 para todos los grupos (Figura 2). La comparación post hoc de las medias de los grupos (Student Newman-Keuls) mostró que los sujetos tratados con NMDA, BLMP 3.0, presentaron latencias de respuesta mayores que los tratados con salina o con BLMP $0.3(P<0.05)$. La Figura 3 muestra el delta de las latencias de respuesta durante los Ensayos 5 y test, para todos los grupos. Esta delta indica el establecimiento del aprendizaje. Un delta mayor indica una pendiente más inclinada de la latencia de respuesta; por tanto, un efecto farmacológico acentuado en el ensayo inicial (Ensayo 5) seguido de una pérdida de efecto en el Ensayo de test. El ANOVA mostró diferencias significativas para el delta de las latencias entre los Ensayos 5 y test $\left(F_{[3,27]}=3.632\right.$; $P=0.025)$. La comparación post hoc de las medias de los grupos, mostró que los sujetos tratados con NMDA presentaron un delta mayor que los demás grupos $(P<0.05)$.

\section{TABLA 1}

Análisis multivariado y significancia con una $\mathrm{p}=$ 0.05 de las sustancias administradas en términos de latencia y errores de la memoria espacial de los sujetos $(\mathrm{N}=31)$ evaluados

Salina Nmda Blmp 0,3 Blmp 3

\begin{tabular}{|c|c|c|c|c|c|}
\hline $\begin{array}{c}\text { Variable } \\
\text { indepen- } \\
\text { diente }\end{array}$ & Grupo & Sig. ${ }^{a}$ & Sig. ${ }^{a}$ & Sig. ${ }^{a}$ & Sig. ${ }^{a}$ \\
\hline \multirow{4}{*}{$\begin{array}{l}\text { Latencia } \\
\text { pruebas }\end{array}$} & SALINA & - & $0.506^{*}$ & $0.685^{*}$ & $0.478 *$ \\
\hline & NMDA & 0.506 & - & $0.777 *$ & $0.997^{*}$ \\
\hline & $\begin{array}{l}\text { BLMP-101 } \\
0,3 \mathrm{ml}\end{array}$ & 0.685 & 0.777 & - & $0.759 *$ \\
\hline & $\begin{array}{l}\text { BLMP-101 } \\
3,0 \mathrm{ml}\end{array}$ & 0.478 & 0.997 & 0.759 & - \\
\hline \multirow{4}{*}{$\begin{array}{l}\text { Errores } \\
\text { prueba }\end{array}$} & SALINA & - & $0.266^{*}$ & $0.569 *$ & $0.857^{*}$ \\
\hline & NMDA & 0.266 & - & $0.555^{*}$ & $0.315^{*}$ \\
\hline & $\begin{array}{l}\text { BLMP-101 } \\
0,3 \mathrm{ml}\end{array}$ & 0.569 & 0.555 & - & $0.674 *$ \\
\hline & $\begin{array}{l}\text { BLMP-101 } \\
3,0 \mathrm{ml}\end{array}$ & 0.857 & 0.315 & 0.674 & - \\
\hline
\end{tabular}

*Diferencias significativas con una $p=0.05$

Basadas en las medias marginales estimadas.

Ajuste para comparaciones múltiples: Diferencia menos significativa (equivalente a la ausencia de ajuste).

Fuente: elaboración propia.

TABLA 2

Estadísticos descriptivos de los grupos evaluados en cuanto a la latencia de la memoria espacial

\begin{tabular}{llccc}
\hline Variables & Grupo & Media & $s$ & $N$ \\
\hline \multirow{4}{*}{$\begin{array}{l}\text { Latencia } \\
\text { prueba }\end{array}$} & SALINA & 36,86 & 29,402 & 7 \\
& NMDA & 56,00 & 55,815 & 7 \\
& BLMP 3 0,3 & 48,13 & 54,315 & 8 \\
& Total & 46,11 & 62,760 & 9 \\
\hline \multirow{5}{*}{ Errores } & SALINA & 3,14 & 50,945 & 31 \\
\hline prueba & NMDA & 5,14 & 2,116 & 7 \\
& BLMP 0,3 & 4,13 & 4,581 & 8 \\
& BLMP 3 & 3,44 & 2,920 & 9 \\
& Total & 3,94 & 3,214 & 31 \\
\hline
\end{tabular}

Fuente: elaboración propia. 
FIGURA 1

Polígono de frecuencias donde se representa la latencia de la memoria espacial en los cuatro grupos

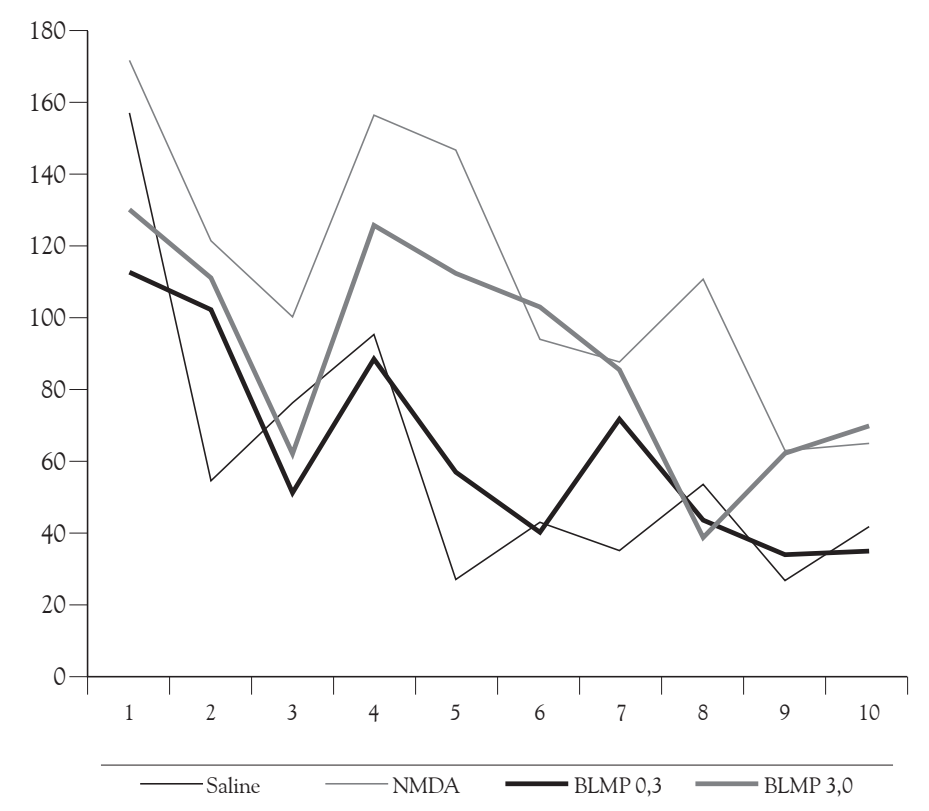

Fuente: elaboración propia.

\section{FiguRA 2}

Polígono de frecuencias donde se representa los errores cometidos por los sujetos de los cuatro grupos en el test de Barnes

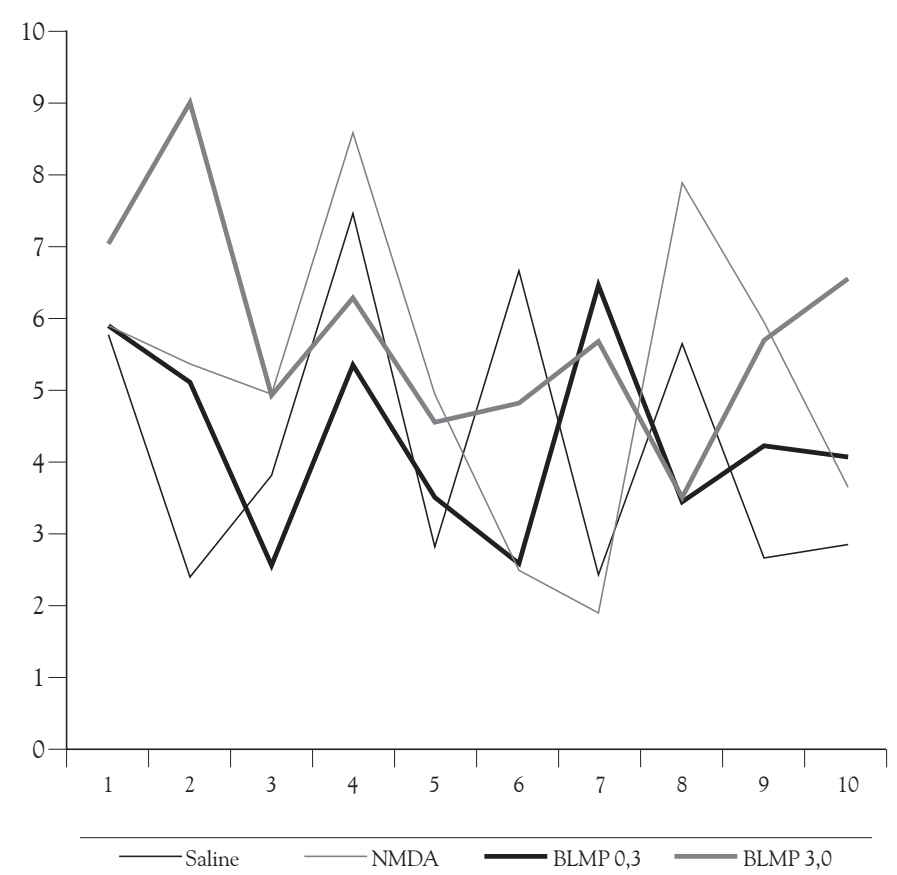

Fuente: elaboración propia. 


\section{Discusión}

El objetivo central del presente trabajo fue evaluar los efectos sobre la memoria de la aplicación intrahipocampal de un péptido desarrollado por modelación computarizada, con una estructura morfológica que le permitiera específicamente ligarse a la subunidad NR2B del complejo del receptor NMDA. Para esto, se utilizaron dos dosis de dicho péptido (BLMP-101) y se comparó su efecto con el de una dosis de NMDA, cuyo efecto es conocido y reportado en la literatura.

Los resultados obtenidos indicaron que los sujetos que recibieron el péptido BLMP-101 en la dosis mayor $(3.0 \mathrm{mg} / \mathrm{kg})$ tuvieron un desempeño parecido al de aquellos que recibieron NMDA en la ejecución del laberinto de Barnes, perjudicándose la adquisición y por tanto interfiriendo con el aprendizaje. Por el contrario, los sujetos que recibieron una dosis menor del péptido $(0,3 \mathrm{mg} /$ $\mathrm{kg}$ ) tuvieron un desempeño parecido al de aquellos que recibieron tratamiento con salina.

Es posible sugerir que el efecto de interferencia del péptido BLMP-101 sobre el aprendizaje de una tarea espacial, sea debido a su papel agonista sobre la subunidad NR2B del complejo del receptor NMDA. De hecho, estudios previos realizados en nuestros laboratorios (datos no publicados) han demostrado que la aplicación del péptido BLMP-101 en la substancia gris periventricular dorsal no induce ninguna de las reacciones de fuga o de congelamiento inducidas con la aplicación de N-metil-D-aspartato, lo cual fue tomado como indicativo de la especificidad de BLMP-101 por la subunidad NR2B, cuya expresión es menor en PAG que en hipocampo (Loftis \& Janowsky, 2002; Tolle et al., 1993) .

Igualmente, la similitud del efecto de interferencia con el aprendizaje del laberinto de Barnes, encontrado en este trabajo, para la aplicación intrahipocampal de BLMP-101 y de NMDA, indica que BLMP 101 posee efecto sobre esta subunidad, cuyo papel en el aprendizaje ha sido clásicamente reportado (Gao et al., 2009). Sin embargo, y por tratarse de una molécula nueva, se requiere mayor investigación sobre sus efectos en otras áreas, que intervienen en el aprendizaje y en otros modelos de memoria.

En cuanto a los resultados histológicos, el análisis de la ramificación dendrítica, se realizó calculando la relación entre el área ocupada por las fibras inmunomarcadas para MAP2 y el área total del campo. Se utilizó un análisis de varianza ANOVA de una vía. Cuando fue necesario, se realizaron comparaciones de las medias de los grupos utilizando el test post hoc Student Newman-Keuls. El nivel de alfa fue estipulado en 0.05. Los cortes tratados con coloración de Nissl, fueron utilizados para seleccionar los animales que entraron en el estudio; animales con cánulas en lugares diferentes al hipocampo ventral, fueron retirados del estudio.

Por su parte, los efectos comportamentales, evidenciados en la Figura 1, muestran los promedios de latencias de respuesta de resolución del laberinto de Barnes para los animales de todos los grupos, para los Ensayos 3 a 10 y el Ensayo de test. En cuanto a los análisis independientes de latencias y número de errores por ensayos, para los Ensayos 4 y 5, en los cuales se observa la inflexión de la curva de aprendizaje, así como la delta entre los ensayos cuatro-test y cinco-test se representan en las figuras siguientes. El ANOVA mostró diferencias significativas $\left(F_{[3,27]}=4.038 ; P=0.017\right)$ para la latencia de respuesta durante el Ensayo 5 para todos los grupos (Figura 2). La comparación post hoc de las medias de los grupos (Student Newman-Keuls) mostró que los sujetos tratados con NMDA, BLMP 3.0 presentaron latencias de respuesta mayores que los tratados con salina o con BLMP $0.3(P<0.05)$.

La Figura 3 muestra el delta de las latencias de respuesta durante los Ensayos 5 y test para todos los grupos. Esta delta indica el establecimiento del aprendizaje. Un delta mayor indica una pendiente más inclinada de la latencia de respuesta, por tanto un efecto farmacológico acentuado en el ensayo inicial (Ensayo 5) seguido de una pérdida de efecto en el Ensayo de test. El ANOVA mostró diferencias significativas para el delta de las latencias entre los Ensayos 5 y test $\left(F_{[3,27]}=3.632 ; P\right.$ $=0.025)$. La comparación post hoc de las medias de los grupos mostró que los sujetos tratados con 
NMDA presentaron un delta mayor que los demás grupos $(P<0.05)$.

En términos generales se presentó un efecto estadísticamente significativo en cuanto a la latencia, cuando se hace tratamiento con BLMP 3. Esto significa que fisiológicamente existe efecto sobre la latencia solo cuando se usa BLMP en la mayor concentración. El tratamiento con BLMP 0.3 se comporta como el control con salina.

\section{Referencias}

Allain, H., Lieury, A., Reymann, J., Martinet, J., Trebon, P., Decombe, R., Bentue-Ferrer, D. \& Gandon, J. M. (1990). Development of memory-improving drugs. Annales de Médecine Interne, 141 (Suppl. 1), 19-25.

Alonso, D., Khalil, Z., Satkunanthan, N. \& Livett, G. (2003). Drugs from the sea: Conotoxins as drug leads for neuropathic pain and other neurological conditions. Mini-Reviews in Medicinal Chemistry, 3 (7), 785-787.

Ault, A. (2004). Ventures with venons. The Scientist, 18 (19), 43-44.

Barnes, C. (1979). Memory deficits associated with senescence: A neurophysiological and behavioral study in the rat. Journal of Comparative and Physiological Psychology, 93 (1), 74-104.

Blandl, T., Warder, S., Prorok, M. \& Castellino, F. (2000). Structure-function relationships of the NMDA receptor antagonist peptide, conantokinR. FEBS Letters, 470 (2), 139-146.

Blandl, T., Zajicek, J., Prorok, M. \& Castellino, F. (2001). Sequence requirements for the N-methyl-D-aspartate receptor antagonist activity of conantokin-R. The Journal of Biological Chemistr, 276 (10), 7391-7396.

Burke, S. N., Chawla, M., Penner, M., Crowell, B., Worley, P., Barnes, C. \& McNaughton, B. (2005). Differential encoding of behavior and spatial context in deep and superficial layers of the neocortex. Neuron, 45, 667-674.

Donevan, S. \& McCabe, R. (2000). Conantokin G is an NR2B-selective competitive antagonist of $\mathrm{N}$ methyl-D-aspartate receptors. Molecular Pharmachology, 58 (3), 614-623.
Echeverry, S. \& Oyuela, R. (2006). Efecto de la aplicación subcutánea del péptido experimental Boost Memory Learning Peptide -BMPL-101, agonista del NMDA, en los procesos de memoria espacial en ratas Wistar -hembras y machos-. Tesis de grado no publicada, Pontificia universidad Javeriana, Bogotá, Colombia.

Bernal, G., Rojas D. \& Oyuela, R. (2006). Evaluación de los efectos de la actividad física y de un péptido sintético experimental en la memoria espacial de ratas Wistar. Tesis de grado no publicada, Pontificia universidad Javeriana, Bogotá, Colombia.

Gentile, M. (1997). Sodium urate arthritis: Effects on the sensory properties of articular afferents in the chicken. Pain, 70, 245-251.

Haack, J., Rivier, J., Parks, T., Mena, E., Cruz, L. \& Olivera, B. (1990). Conantokin-T. A gammacarboxyglutamate containing peptide with $\mathrm{N}$ methyl-d-aspartate antagonist activity. The Journal of Biological Chemistry, 265 (11), 6025-6029.

Hammerland, L., Olivera, B. \& Yoshikami, D. (1992). Conantokin-G selectively inhibits N-methyl-Daspartate-induced currents in Xenopus oocytes injected with mouse brain mRNA. European Journal of Pharmacology, 226 (3), 239-244.

Heresco-Levy, U. \& Javitt, D. (1998). The role of Nmethyl-D-aspartate (NMDA) receptor-mediated neurotransmission in the pathophysiology and therapeutics of psychiatric syndromes. European Journal of Pharmacology, 8 (2), 141-152.

Johnson, G. \& Jope, R. (1992): The role of microtubuleassociated protein 2 (MAP-2) in neuronal growth, plasticity, and degeneration. Journal of Neuroscience Research, 33, 505-512.

Lareo, L., R., Oyuela, L., Muñoz, L., Morales, S., Echeverri, A., Uribe, O. et al. (2005). Diseño y evaluación de un péptido para incrementar el aprendizaje y la memoria en ratas. Revista de la Asociación Colombiana de Ciencias Biológicas, 16, 107-116.

Loftis, J. \& Janowsky, A. (2002). The N-methyl-Daspartate receptor subunit NR2B: Localization, functional properties, regulation, and clinical implications. Pharmacology and Therapeutics, 97, 55-85.

Narahashi, T., Moriguchi, S., Zhao, X., Marszalec, W. \& Yeh, J. (2004). Mechanisms of action of cognitive 
enhancers on neuroreceptors. Biological and Pharmaceutical Bulletin, 27 (11), 1701-1706.

Nielsen, K., Skjaerbaek, N., Dooley, M., Adams, D., Mortensen, M., Dodd, P. et al. (1999). Structureactivity studies of conantokins as human $\mathrm{N}$-methyl-D-aspartate receptor modulators. The Journal of Biological Chemistry, 42 (3), 415-26.

Oyuela, R., Lareo, L., Muñoz, L., Morales, L., Echeverry, S., Uribe, A. et al. (2004). Estudio preliminar sobre el efecto en el aprendizaje y la memoria espacial de un péptido sintético en ratas. Psicología desde el Caribe, 13, 1-14.

Paxinos, G. \& Watson, C. (1997). The Rat Brain in Stereotaxic Coordinates, New York: Academic Press, Paper Back.

Randall, L. \& Selitto, J. (1957). A method for measurement of analgesic activity on inflamed tissue. Archives: International Pharmacology Therapy, 111, $409-419$.

Riedel, G., Platt, B. \& Micheau, J. (2003). Glutamate receptor function in learning and memory. Behavioural Brain Research, 140 (1-2), 1-47.

Santacruz, M. del P., Oyuela, R., Brínez, J. A., Lareo, L. \& Echeverri, S. L. (2008). Efectos sobre la actividad nociceptiva de la rata de un péptido nootrópico sintético. Revista Latinoamericana de Psicología, 40 (1), 97-109.

Tang, Y., Shimizu, E., Dube, G., Rampon, C., Kerchner, G., Zhuo, M. et al. (1999). Genetic enhancement of learning and memory in mice. Nature, 401 , 63-69.

Tang, Y., Wang, H., Feng, R., Kyin, M. \& Tsien, J. (2001). Differential effects of enrichment on learning and memory function in NR2B transgenic mice. Neuropharmacology, 41 (6), 779-790.

Tolle, T., Berthele, A., Zieglgansberger, W., Seeburg, P. H. \& Wisden, W. (1993). The differential expression of 16 NMDA and non-NMDA receptor subunits in the rat spinal cord and in periaqueductal gray. Journal of Neuroscience, 13, 5009-5028.

Tsien, J. \& Ezzell, C. (2000). Learning, memory, genetic engineering, rodents. Scientific American, 282 (4), 62-67.

Van Reekum, R., Black, S., Conn, D. \& Clarke, D. (1997). Cognition-enhancing drugs in dementia: A guide to the near future. Canadian Journal of Psychiatry, 42 (Suppl. 1), 35-50. 